\title{
Improved SPH methods for simulating free surface flows of viscous fluids
}

\author{
Jiannong Fang ${ }^{\mathrm{a}, *}$, Aurèle Parriaux ${ }^{\mathrm{a}}$, Martin Rentschler ${ }^{\mathrm{b}}$, \\ Christophe Ancey ${ }^{\mathrm{b}}$ \\ ${ }^{a}$ Engineering and Environmental Geology Laboratory (GEOLEP), Institute of \\ infrastructures, resources and environment, Swiss Federal Institute of Technology \\ Lausanne, EPFL-ICARE, CH-1015 Lausanne, Switzerland \\ ${ }^{\mathrm{b}}$ Environmental Hydraulics Laboratory (LHE), Institute of infrastructures, \\ resources and environment, Swiss Federal Institute of Technology Lausanne, \\ EPFL-ICARE, CH-1015 Lausanne, Switzerland
}

\begin{abstract}
In this paper we present two enhanced variants of the smoothed particle hydrodynamics (SPH) method for the numerical simulation of free surface flows of viscous fluids. Improvements are achieved by deriving a new set of general discrete SPH-like equations under an energy-based framework and applying a corrected (high-order) or coupled particle approximation scheme for function derivatives. By doing so, we ensure that the enhanced variants retain the conservative nature of SPH which is important for the stability of long-term simulations. Among various corrected approximations, we here implement the one obtained by the so-called finite particle method (FPM) within the framework to produce a higher-order SPH method which conserves both linear and angular momentums. In order to improve the efficiency of the higher-order variant, a coupled approach with the idea of using the SPH approximation for the interior particles and the FPM approximation for the exterior particles is also proposed and tested in this paper. Three prototype tests concerning free deformation of a viscous fluid patch with free surface are presented with comparisons between different methods to demonstrate the performance of the two proposed methods. Numerical results show that both the higher-order version using FPM and the coupled version using FPM/SPH outperform the original version of $\mathrm{SPH}$ in respect of accuracy and stability.
\end{abstract}

Key words: Meshless/meshfree method; Smoothed Particle Hydrodynamics; Finite particle method; Viscous fluids; Free surface flows

* Corresponding author. Tel.: ++41 21693 2357. Fax: ++41216936330.

Email address: jiannong.fang@epfl.ch (Jiannong Fang). 


\section{Introduction}

Recently, in the general area of computational mechanics there is a growing interest in developing so-called meshless/meshfree methods or particle methods as alternatives to traditional grid-based methods such as finite difference methods and finite element methods. Among the various meshfree and particle methods, Smoothed Particle Hydrodynamics ( $\mathrm{SPH}$ ) is the longest established and is quickly approaching a mature stage. The SPH method was originally developed for astrophysical applications $[18,28]$. Since its invention, it has been extensively studied, extended and applied in many areas such as the dynamic response of elasto-plastic materials [2,23,24], free surface flows [31], viscous flows $[17,36,45,46]$, solid friction [29], incompressible fluids [10,42], heat transfer [7], multi-phase flows $[32,37]$, geophysical flows $[1,8,38]$, turbulence modeling [34], viscoelastic flows [13,14], and free surface viscoelastic flows [16]. For more information on the SPH method, we refer the reader to the book by Liu and Liu [25] and the most recent review of the method by Monaghan [35].

$\mathrm{SPH}$ is a Lagrangian meshless method in which the problem to be solved is discretized using particles that are free to move rather than by fixed grids or meshes. The governing PDEs are converted into equations of motion for these particles. SPH has several advantages over grid-based methods. Amongst these we highlight the following:

- It handles convection dominated flows and large deformation problems very well due to its Lagrangian and adaptive nature;

- Complex free surfaces, including those with fragmentation, are modeled naturally without the need of any form of explicit surface tracking;

- Complicated physics such as multi-phase, realistic equations of state, compressibility, radiation and solidification can be added easily;

- It handles easily complex geometries in two and three dimensions;

- Programming for complex problems is an easy task compared with other methods such as finite element methods.

Another unique and attractive characteristic of the SPH method is the harmonious combination of the Lagrangian formulation and particle approximation, which makes the SPH particles not only function as interpolation points like the particles in other mesh-free methods but also carry material properties.

Yet, SPH suffers from several drawbacks: low accuracy; tensile instability; difficulty in enforcing essential boundary conditions. This paper mainly addresses the first of these deficiencies, which originates from the particle approximation procedure adopted in SPH (see Section 3.3 for details). Where appropriate, tensile instability which is closely related to the problem of low accuracy, will also be discussed. Various methods have been developed to improve the accu- 
racy of the conventional SPH. The symmetrization and anti-symmetrization in some SPH formulations [30] were first attempted to improve the accuracy of the SPH particle approximation. A reproducing kernel particle method was developed by Liu et al. [27] to reproduce consistency conditions by correcting the smoothing function which is a key component in particle approximation. Johnson and Beissel [22] proposed a normalized smoothing function algorithm. Dilts $[11,12]$ introduced the moving least square approximation into SPH computations. Using Taylor series expansion, Chen and Beraun [6] developed a corrected smoothed particle hydrodynamics method for nonlinear dynamic problems. Their work was followed recently by Liu et al. [26] who proposed the so-called finite particle method (FPM) and applied it to viscous fluid flows. Zhang and Batra [47] applied a similar idea to transient problems in elastic dynamics and heat conduction. Most of the previous contributions attempting to improve SPH have focused on replacing directly the existing SPH particle approximations to functions and their derivatives by corrected approximations with polynomial consistency enforced. As a consequence, angular momentum and/or linear momentum are usually not strictly conserved by the discrete equations and the long-time simulations are potentially unstable. Differently, Bonet and Lok [3] presented a corrected SPH formulation using a variational framework with the advantage of preserving both linear and angular momentum.

The purpose of this paper is to derive and test a new set of discrete conservative hydrodynamics equations for the simulation of free surface flows of viscous fluids with improved accuracy and stability by using a corrected or coupled particle approximation within an energy-based framework. It turns out that the energy-based formulation here is consistent with the variational formulation [3] and the so-obtained new set of discrete equations is a bit more general than that in the latter. The outline of the paper is as follows. In the next section, the partial differential equations (PDEs) governing the flow of a viscous fluid are outlined. In Section 3 we first give brief introductions on the two particle approximations, namely, SPH and FPM, and then derive a general set of discrete hydrodynamics equations from the PDEs by following an energy-based approach [15] from which conservation of linear momentum is guaranteed and conservation of angular momentum is also ensured when a particle approximation of first-order consistency is used. After that, a conventional SPH formulation is revisited in the energy-based framework and two new particle hydrodynamics methods are obtained by actualizing the general set of discrete hydrodynamics equations with the FPM particle approximation and a coupled FPM/SPH particle approximation, respectively. In Section 4, three prototype tests concerning free deformation of a viscous fluid patch are used to discuss the performance of various methods. The paper ends up with concluding remarks in Section 5. 


\section{Governing equations}

In a Lagrangian frame, the continuity equation and $\alpha$-th projection of the momentum balance equation for a viscous fluid may be written as

$$
\begin{gathered}
\frac{D \rho}{D t}=-\rho \frac{\partial v^{\beta}}{\partial x^{\beta}}, \\
\rho \frac{D v^{\alpha}}{D t}=-\frac{\partial p}{\partial x^{\alpha}}+\frac{\partial \tau^{\alpha \beta}}{\partial x^{\beta}},
\end{gathered}
$$

where $\rho$ denotes the fluid density , $v^{\beta}$ the $\beta$-th component of the fluid velocity, $p$ the isotropic pressure, $\tau^{\alpha \beta}$ the $(\alpha, \beta)$-th component of the viscous stress tensor. If neglecting the heat flux and the body force, the energy equation can be written as follows.

$$
\rho \frac{D e}{D t}=\phi-p \frac{\partial v^{\beta}}{\partial x^{\beta}}
$$

where $e$ is the internal energy per unit mass. The dissipation function $\phi$ is defined by

$$
\phi=\tau^{\alpha \beta} d^{\beta \alpha}
$$

where the traceless symmetric velocity gradient tensor is

$$
d^{\alpha \beta}=\frac{1}{2}\left[\frac{\partial v^{\alpha}}{\partial x^{\beta}}+\frac{\partial v^{\beta}}{\partial x^{\alpha}}\right]-\frac{1}{\lambda}\left[\frac{\partial v^{\gamma}}{\partial x^{\gamma}}\right] \delta^{\alpha \beta}
$$

with $\delta^{\alpha \beta}=1$ if $\alpha=\beta$ and $\delta^{\alpha \beta}=0$ if $\alpha \neq \beta$, and $\lambda$ the spatial dimension. The viscous stress tensor is given by

$$
\tau^{\alpha \beta}=2 \eta d^{\alpha \beta}
$$

with the shear viscosity denoted by $\eta$ and the bulk viscosity assumed to be null. In the above equations, the spatial coordinates $x^{\alpha}$ and time $t$ are the independent variables and $D / D t$ is the material (total) derivative defined in a fixed Eulerian frame by

$$
\frac{D}{D t}=\frac{\partial}{\partial t}+v^{\beta} \frac{\partial}{\partial x^{\beta}}
$$

Note that the Einstein summation convention (the summation is taken over repeated indices) is applied in this paper to $\alpha$ or $\beta$ but not to $i$ or $j$.

In the standard SPH method for solving compressible flows, the pressure is calculated by the local density and internal energy through the equation of state. The real fluids such as water are compressible, but with a speed of sound $c$ which is very much greater than the speed of bulk flow. Hence using the actual equation of state of the fluid will lead to prohibitive time step that are extremely small. It can be shown [31] that the density variation is 
proportional to the square of the Mach number $M^{2}(M \equiv V / c$, where $\mathrm{V}$ is a typical reference velocity). For fluids like water, with $c \sim 10^{3} \mathrm{~m} / \mathrm{s}$, the Mach number is extremely small, and it is customary to approximate the fluid by a theoretically incompressible fluid. Though it is possible to include the constraint of the constant density into the SPH formulations, the resultant equations are too cumbersome. Many previous works using the SPH method for the simulation of fluid flows adopted a different approach [31], in which the real fluid is approximated by an artificial fluid which is more compressible than the real fluid. The artificial fluid has a specially designed equation of state with a speed of sound which is still much larger than the speed of bulk flow and therefore has very small density fluctuations. In this paper, we follow this approach by using the following equation of state:

$$
p(\rho)=c^{2}\left(\rho-\rho_{0}\right),
$$

where $\rho_{0}$ is a reference density. A suitable choice of $c$ can produce the desired density variation. For the simulations performed in this paper, we control the density variation to within $1 \%$ so that the behavior of the artificial compressible fluid is sufficiently close to the real fluid.

\section{Discretisation}

\subsection{SPH method}

The interpolation process of SPH is based on the integral representation of a field function $f(\boldsymbol{x})$,

$$
\langle f(\boldsymbol{x})\rangle=\int_{\Omega} f\left(\boldsymbol{x}^{\prime}\right) W\left(\boldsymbol{x}-\boldsymbol{x}^{\prime}, h\right) d \boldsymbol{x}^{\prime},
$$

where $W$ is the so-called smoothing function or kernel function and $h$ is the smoothing length defining the influence area of $W$. The integral representation converges when $\mathrm{W}$ satisfies the following conditions:

$$
\int_{\Omega} W\left(\boldsymbol{x}-\boldsymbol{x}^{\prime}, h\right) d \boldsymbol{x}^{\prime}=1
$$

and

$$
\lim _{h \rightarrow 0} W\left(\boldsymbol{x}-\boldsymbol{x}^{\prime}, h\right)=\delta\left(\boldsymbol{x}-\boldsymbol{x}^{\prime}\right) .
$$

Moreover, to be valid, it is often required that

$$
W\left(\boldsymbol{x}-\boldsymbol{x}^{\prime}, h\right)>0 \quad \text { over } \quad \Omega
$$

and

$$
W\left(\boldsymbol{x}-\boldsymbol{x}^{\prime}, h\right)=0 \quad \text { when } \quad\left|\boldsymbol{x}-\boldsymbol{x}^{\prime}\right|>k h,
$$


where $k$ is a constant which is a measure of the effective (non-zero) area of the smoothing function centered at a point having position vector $\boldsymbol{x}$. This effective area is called the support domain of the point $\boldsymbol{x}$. Using the compact condition (13), integration over the entire problem domain is localized as the integration over the support domain of a given point. If the smoothing function $W$ is an even function in $\boldsymbol{x}$, by using the Taylor series expansion of $f\left(\boldsymbol{x}^{\prime}\right)$ around $\boldsymbol{x}$, it can be shown that, with the normalization condition (10), the integral representation of $f(\boldsymbol{x})$ is of second order $\left(\mathcal{O}\left(h^{2}\right)\right)$ accuracy. However, this is true only for interior regions. For boundary regions, the SPH integral approximation does not have second order accuracy.

The continuous integral representation (9) can be discretized as a summation over all particles in the support domain to give the so called particle approximation as follows:

$$
\langle f(\boldsymbol{x})\rangle \simeq \sum_{j} \frac{m_{j}}{\rho_{j}} f_{j} W\left(\boldsymbol{x}-\boldsymbol{x}_{j}, h\right)
$$

where $m_{j}$ and $\rho_{j}$ are the mass and density of the particle $j$ respectively $\left(m_{j} / \rho_{j}\right.$ gives the volume $V_{j}$ associated with the particle $j$ ) and $j=1,2, \ldots, N$ in which $N$ is the number of particles within the support domain of particle $\boldsymbol{x}$. Note that the discretized form of the normalization condition $\sum_{j} W\left(\boldsymbol{x}-\boldsymbol{x}_{j}, h\right) V_{j}=1$ is valid only for some simple cases, but not valid for randomly distributed particles or particles close to the boundaries. Hence the SPH particle approximation for a function does not have zero-th order consistency even for particles in interior regions i.e. it does not exactly reproduce a constant (0-th order polynomial) function. However, several numerical studies indicate that SPH is at least first order accurate when the particle set maintains its regularity and the overlap property.

The particle approximation for the spatial derivative $\partial f(\boldsymbol{x}) / \partial \boldsymbol{x}$ can be obtained simply by substituting $f(\boldsymbol{x})$ with $\partial f(\boldsymbol{x}) / \partial \boldsymbol{x}$ in Eq. (9). After integrating by parts and using the divergence theorem, this leads to

$$
\left\langle\frac{\partial f(\boldsymbol{x})}{\partial \boldsymbol{x}}\right\rangle=\int_{S} f\left(\boldsymbol{x}^{\prime}\right) W\left(\boldsymbol{x}-\boldsymbol{x}^{\prime}, h\right) \boldsymbol{n} d S-\int_{\Omega} f\left(\boldsymbol{x}^{\prime}\right) \frac{\partial W\left(\boldsymbol{x}-\boldsymbol{x}^{\prime}, h\right)}{\partial \boldsymbol{x}^{\prime}} d \boldsymbol{x}^{\prime} .
$$

The first term on the right-hand side of Eq. (15) is the so-called residual boundary integral. In the original SPH approximation, it is neglected. By doing so and using summation over particles to replace continuous integration in the second term in Eq. (15) as was done in deriving Eq. (14) from Eq. (9), we obtain

$$
\left\langle\frac{\partial f(\boldsymbol{x})}{\partial \boldsymbol{x}}\right\rangle \simeq-\sum_{j} \frac{m_{j}}{\rho_{j}} f_{j} \frac{\partial W\left(\boldsymbol{x}-\boldsymbol{x}_{j}, h\right)}{\partial \boldsymbol{x}_{j}} .
$$

It is important to note that the residual boundary integral is zero when the support domain of a given particle is located within the problem domain due 
to the compact condition (13) for $W$. However, it is normally no longer zero when the support domain is truncated by the boundary of the problem domain. The latter case is closely related to the problem of particle deficiency near or on the boundary. For particles near or on the boundary, only particles inside the boundary contribute to the SPH summation for the gradient calculation and there is no contribution from outside. The one-sided contribution causes inaccurate solutions. A natural attempt at solving the problem by reverting to the original integral estimate and using the boundary conditions through the residual boundary integration failed for some simple problems. So, common practice in solving the problem of particle deficiency is still to use the various techniques based on virtual (or, ghost) particles [36,41,45].

The particle approximation for a function and its derivatives at particle $i$ can finally be written in condensed form as

$$
\begin{gathered}
f_{i}=\sum_{j} \frac{m_{j}}{\rho_{j}} f_{j} W_{i j} \\
\left(\frac{\partial f}{\partial \boldsymbol{x}}\right)_{i}=\sum_{j} \frac{m_{j}}{\rho_{j}}\left(f_{j}-f_{i}\right) \frac{\partial W_{i j}}{\partial \boldsymbol{x}_{i}}
\end{gathered}
$$

where $W_{i j}=W\left(\boldsymbol{x}_{i}-\boldsymbol{x}_{j}, h\right)$ and $\partial W_{i j} / \partial \boldsymbol{x}_{i}=\partial W\left(\boldsymbol{x}_{i}-\boldsymbol{x}_{j}, h\right) / \partial \boldsymbol{x}_{i}$. It should be noted that the gradient $\partial W_{i j} / \partial \boldsymbol{x}_{i}$ is calculated with respect to $\boldsymbol{x}_{i}$ not $\boldsymbol{x}_{j}$ as in Eq. (16), so the negative sign in Eq. (16) is removed in Eq. (18). Eq. (18) also introduces " $-f_{i}$ " into the discrete particle approximation in order to ensure that the derivatives of a constant function vanish. Because $W_{i j}$ is a function of the distance $r=\left|\boldsymbol{x}_{i}-\boldsymbol{x}_{j}\right|$ between the particles $i$ and $j$, we have

$$
\frac{\partial W_{i j}}{\partial \boldsymbol{x}_{i}}=\left(\frac{\boldsymbol{x}_{i}-\boldsymbol{x}_{j}}{r}\right) \frac{\partial W_{i j}}{\partial r}
$$

and therefore

$$
\frac{\partial W_{j i}}{\partial \boldsymbol{x}_{j}}=-\frac{\partial W_{i j}}{\partial \boldsymbol{x}_{i}}
$$

The smoothing function is one of the most important ingredients of the SPH method. Its choice is related to the accuracy, efficiency, and stability of the resulting algorithm. In the literature many possible forms for it have been proposed ranging from Gaussian functions to spline functions with the compact condition. In this paper we chose the quintic spline function as the smoothing 
function. Writing $q=r / h$ and choosing $k$ in Eq. (13) equal to 3, it reads

$$
W_{i j}=W(r, h)=w_{0} \times \begin{cases}(3-q)^{5}-6(2-q)^{3}+15(1-q)^{5}, & 0 \leq q<1 \\ (3-q)^{5}-6(2-q)^{3}, & 1 \leq q<2 \\ (3-q)^{5}, & 2 \leq q<3 \\ 0, & q \geq 3\end{cases}
$$

where the normalization factor $w_{0}=7 /\left(478 \pi h^{2}\right)$ in two-dimensional problems.

In SPH method, the velocity gradients are calculated by using the formula (18) to give

$$
\left(\frac{\partial v^{\alpha}}{\partial x^{\beta}}\right)_{i}=\sum_{j} \frac{m_{j}}{\rho_{j}}\left(v_{j}^{\alpha}-v_{i}^{\alpha}\right) \frac{\partial W_{i j}}{\partial x_{i}^{\beta}} .
$$

\subsection{Finite particle method}

Following the work of Chen and Beraun [6], Liu et al. [26] proposed a set of correction formulas for both the SPH kernel function and the derivatives of the SPH kernel function, which they called finite particle method. A similar work was independently done by Zhang and Batra [47]. We here briefly describe the finite particle method as below.

A sufficiently smooth function $f(\boldsymbol{x})$ at point $\boldsymbol{x}$ can be expressed by a Taylor series expansion to first order as follows:

$$
f(\boldsymbol{x})=f_{i}+\left(x^{\alpha}-x_{i}^{\alpha}\right) f_{i, \alpha},
$$

where $f_{i}$ and $f_{i, \alpha}$ are defined as

$$
\begin{aligned}
f_{i} & =f\left(\boldsymbol{x}_{i}\right), \\
f_{i, \alpha} & =\left(\frac{\partial f}{\partial x^{\alpha}}\right)_{i} .
\end{aligned}
$$

Multiplying both side of Eq. (23) with a weight function $\varphi\left(\boldsymbol{x}-\boldsymbol{x}_{i}, h\right)$ with a compact support and integrating over the problem space $\Omega$ can yield the following equation:

$\int_{\Omega} f(\boldsymbol{x}) \varphi\left(\boldsymbol{x}-\boldsymbol{x}_{i}, h\right) d \boldsymbol{x}=f_{i} \int_{\Omega} \varphi\left(\boldsymbol{x}-\boldsymbol{x}_{i}, h\right) d \boldsymbol{x}+f_{i, \alpha} \int_{\Omega}\left(x^{\alpha}-x_{i}^{\alpha}\right) \varphi\left(\boldsymbol{x}-\boldsymbol{x}_{i}, h\right) d \boldsymbol{x}$.

Like SPH, the integrals in the above equation can be numerically approximated 
by summation over the particles surrounding point $\boldsymbol{x}_{i}$, that gives

$\sum_{j} f\left(\boldsymbol{x}_{j}\right) \varphi\left(\boldsymbol{x}_{j}-\boldsymbol{x}_{i}, h\right) V_{j}=f_{i} \sum_{j} \varphi\left(\boldsymbol{x}_{j}-\boldsymbol{x}_{i}, h\right) V_{j}+f_{i, \alpha} \sum_{j}\left(x_{j}^{\alpha}-x_{i}^{\alpha}\right) \varphi\left(\boldsymbol{x}_{j}-\boldsymbol{x}_{i}, h\right) V_{j}$.

This equation can be rewritten in the form of matrix equation as:

$$
A_{i}^{m n} F_{i}^{n}=B_{i}^{m}
$$

where

$$
\begin{gathered}
F_{i}^{n}=\left[f_{i}, f_{i, \alpha}\right]^{\mathrm{T}}, \\
B_{i}^{m}=\sum_{j} f\left(\boldsymbol{x}_{j}\right) \varphi^{m}\left(\boldsymbol{x}_{j}-\boldsymbol{x}_{i}, h\right) V_{j},
\end{gathered}
$$

and

$$
A_{i}^{m n}=\left[\sum_{j} \varphi^{m}\left(\boldsymbol{x}_{j}-\boldsymbol{x}_{i}, h\right) V_{j}, \quad \sum_{j}\left(x_{j}^{\alpha}-x_{i}^{\alpha}\right) \varphi^{m}\left(\boldsymbol{x}_{j}-\boldsymbol{x}_{i}, h\right) V_{j}\right] .
$$

Corresponding to one-, two-, and three-dimensional cases, there are 1 function value, and 1,2 and 3 first order derivatives that will be approximated by solving the matrix equation (28). It is clear that $m$ is from 1 to 2,3 , and 4 respectively corresponding to one-, two-, and three-dimensional cases and therefore a set of weight functions $\varphi^{m}\left(\boldsymbol{x}_{j}-\boldsymbol{x}_{i}, h\right)$ needs to be determined. Since the purpose of this paper is to improve SPH method, the kernel function in $\mathrm{SPH}$ and its derivatives are also used in FPM as the weight functions. For two-dimensional case, we have

$$
\begin{gathered}
\varphi^{1}\left(\boldsymbol{x}_{j}-\boldsymbol{x}_{i}, h\right)=W_{i j}, \\
\varphi^{2}\left(\boldsymbol{x}_{j}-\boldsymbol{x}_{i}, h\right)=\frac{\partial W_{i j}}{\partial x_{i}^{1}}, \\
\varphi^{3}\left(\boldsymbol{x}_{j}-\boldsymbol{x}_{i}, h\right)=\frac{\partial W_{i j}}{\partial x_{i}^{2}},
\end{gathered}
$$

where $W_{i j}$ is given in Eq. (21). Solving the local matrix equation (28), the function value as well as the first derivatives at particle $i$ can be calculated as

$$
\begin{gathered}
f_{i}=\sum_{j} \frac{m_{j}}{\rho_{j}} f_{j} \widetilde{W_{i j}} \\
\left(\frac{\partial f}{\partial \boldsymbol{x}}\right)_{i}=\sum_{j} \frac{m_{j}}{\rho_{j}} f_{j} \frac{\widetilde{\partial W_{i j}}}{\partial \boldsymbol{x}_{i}},
\end{gathered}
$$

where $V_{j}$ is replaced by $m_{j} / \rho_{j}$ and the corrected kernel function $\widetilde{W_{i j}}$ and its 
derivatives $\frac{\widetilde{\partial W_{i j}}}{\partial \boldsymbol{x}_{i}}$ are given by

$$
\left(\begin{array}{c}
\widetilde{W_{i j}} \\
\frac{\partial W_{i j}}{\partial x_{i}^{1}} \\
\frac{\partial W_{i j}}{\partial x_{i}^{2}}
\end{array}\right)=\left(\begin{array}{ccc}
\sum_{j} W_{i j} V_{j} & \sum_{j}\left(x_{j}^{1}-x_{i}^{1}\right) W_{i j} V_{j} & \sum_{j}\left(x_{j}^{2}-x_{i}^{2}\right) W_{i j} V_{j} \\
\sum_{j} \frac{\partial W_{i j}}{\partial x_{i}^{1}} V_{j} & \sum_{j}\left(x_{j}^{1}-x_{i}^{1}\right) \frac{\partial W_{i j}}{\partial x_{i}^{1}} V_{j} \sum_{j}\left(x_{j}^{2}-x_{i}^{2}\right) \frac{\partial W_{i j}}{\partial x_{i}^{1}} V_{j} \\
\sum_{j} \frac{\partial W_{i j}}{\partial x_{i}^{2}} V_{j} & \sum_{j}\left(x_{j}^{1}-x_{i}^{1}\right) \frac{\partial W_{i j}}{\partial x_{i}^{2}} V_{j} & \sum_{j}\left(x_{j}^{2}-x_{i}^{2}\right) \frac{\partial W_{i j}}{\partial x_{i}^{2}} V_{j}
\end{array}\right)^{-1}\left(\begin{array}{c}
W_{i j} \\
\frac{\partial W_{i j}}{\partial x_{i}^{1}} \\
\frac{\partial W_{i j}}{\partial x_{i}^{2}}
\end{array}\right) .
$$

Again, like in SPH, the velocity gradients are calculated by FPM as

$$
\left(\frac{\partial v^{\alpha}}{\partial x^{\beta}}\right)_{i}=\sum_{j} \frac{m_{j}}{\rho_{j}}\left(v_{j}^{\alpha}-v_{i}^{\alpha}\right) \frac{\partial \widetilde{W}_{i j}}{\partial x_{i}^{\beta}} .
$$

\subsection{An energy-based framework}

In this section, we apply an energy-based approach to build a new set of discrete hydrodynamics equations for which any particle approximations (corrected or not) can be adopted. The energy-based approach has been proposed for the first time by Español and Revenga [15] to derive the so-called smoothed dissipative particle dynamics with the additional aid of the GENERIC formalism $[19,39]$.

In this energy-based framework, the fluid is discretised into a finite number of "particles", which possess individual material properties and move according to the governing equations. The relevant physical quantities on each particle are calculated by an interpolation process over neighboring particles. Every particle is regarded as a moving thermodynamic subsystem with a position $\boldsymbol{x}_{i}$, velocity $\boldsymbol{v}_{i}$, constant mass $m_{i}$, density $\rho_{i}$, volume $V_{i}=m_{i} / \rho_{i}$, and the internal energy $E_{i}=m_{i} e_{i}$.

A general discrete model for Eqs. (1)-(3) can be written as

$$
\begin{gathered}
\dot{\rho}_{i}=-\rho_{i}\left(\frac{\partial v^{\beta}}{\partial x^{\beta}}\right)_{i}, \\
\dot{v}_{i}^{\alpha}=-\frac{1}{\rho_{i}}\left(\frac{\partial p}{\partial x^{\alpha}}\right)_{i}+\frac{1}{\rho_{i}}\left(\frac{\partial \tau^{\alpha \beta}}{\partial x^{\beta}}\right)_{i}, \\
\dot{E}_{i}=V_{i} \phi_{i}-V_{i} p_{i}\left(\frac{\partial v^{\beta}}{\partial x^{\beta}}\right)_{i} .
\end{gathered}
$$

The total energy of the particle system is given by

$$
E=\sum_{i}\left[\frac{m_{i}}{2} v_{i}^{\alpha} v_{i}^{\alpha}+E_{i}\right]
$$


Using Eq. (3), we can show that its time derivative is

$$
\dot{E}=\sum_{i}\left[m_{i} \dot{v}_{i}^{\alpha} v_{i}^{\alpha}-V_{i} p_{i}\left(\frac{\partial v^{\beta}}{\partial x^{\beta}}\right)_{i}+V_{i} \phi_{i}\right] .
$$

To obtain a discrete approximation of the pressure gradient in the momentum equation, we here consider first an inviscid fluid. In this case, with the help of Eq. (40), Eq. (43) becomes

$$
\dot{E}=\sum_{i}\left[P_{i}^{\alpha} v_{i}^{\alpha}-V_{i} p_{i}\left(\frac{\partial v^{\beta}}{\partial x^{\beta}}\right)_{i}\right],
$$

where

$$
P_{i}^{\alpha}=-V_{i}\left(\frac{\partial p}{\partial x^{\alpha}}\right)_{i}
$$

is the $\alpha$-th component of the force exerted on the particle $i$ due to the pressure gradient. The velocity gradients can be formally approximated as

$$
\left(\frac{\partial v^{\alpha}}{\partial x^{\beta}}\right)_{i}=\sum_{j}\left(v_{j}^{\alpha}-v_{i}^{\alpha}\right) C_{i j}^{\beta},
$$

where $C_{i j}^{\beta}$ are interpolation coefficients for a given particle approximation, for example, SPH and FPM introduced in sections 3.1 and 3.2, respectively.

We now introduce the Euler's homogeneous function theorem, which states that a function $f(\boldsymbol{x})=f\left(x_{1}, x_{2}, \ldots, x_{n}\right)$ that is homogeneous of degree $k$ has first order partial derivatives which satisfy

$$
\sum_{i=1}^{n} x_{i} \frac{\partial f(\boldsymbol{x})}{\partial x_{i}}=k f(\boldsymbol{x})
$$

We say that $f(\boldsymbol{x})$ is homogeneous of degree $k$ if the equation

$$
f(a \boldsymbol{x})=a^{k} f(\boldsymbol{x})
$$

holds for a scalar $a$. Denoting the second summation term in the right hand side of Eq. (44) as П, i.e.,

$$
\Pi=\sum_{i} V_{i} p_{i}\left(\frac{\partial v^{\beta}}{\partial x^{\beta}}\right)_{i}
$$

it is then straightforward to show that the potential function $\Pi$ with the velocity gradients expressed by Eq. (46) is a homogeneous function of degree $k=1$ with respect to the variables $v_{i}^{\alpha}(\alpha=1,2,3 ; i=1, \ldots, n)$. Therefore, by applying the Euler's homogeneous function theorem to $\Pi$, Eq. (44) can be 
transformed to

$$
\dot{E}=\sum_{i} v_{i}^{\alpha}\left[P_{i}^{\alpha}-\frac{\partial \Pi}{\partial v_{i}^{\alpha}}\right],
$$

which suggests that a suitable expression for $P_{i}^{\alpha}$ is given by

$$
P_{i}^{\alpha}=\frac{\partial \Pi}{\partial v_{i}^{\alpha}}
$$

which leads to energy conservation, i.e., $\dot{E}=0$. After some differential operations, we finally obtain

$$
P_{i}^{\alpha}=\sum_{j} V_{j} p_{j} C_{j i}^{\alpha}-\sum_{j} V_{i} p_{i} C_{i j}^{\alpha}
$$

Similarly, to derive discrete versions for the gradients of viscous stresses, we resort to an energy consideration that friction forces should produce an increase of internal energy in order to conserve total energy, i.e., the work done by the friction forces on the particles should be equal to the energy dissipated. With this idea and the help of Eqs. (40) and (43), and note that Eq. (44) is zero with the specific choice of $P_{i}^{\alpha}$ given before, we obtain

$$
\sum_{i}\left[F_{i}^{\alpha} v_{i}^{\alpha}+V_{i} \phi_{i}\right]=0
$$

where

$$
F_{i}^{\alpha}=V_{i}\left(\frac{\partial \tau^{\alpha \beta}}{\partial x^{\beta}}\right)_{i}
$$

is the $\alpha$-th component of the friction force exerted on the particle $i$ due to the divergence of viscous stresses and the viscous dissipation function $\phi_{i}$ is evaluated as

$$
\phi_{i}=2 \eta\left(d^{\alpha \beta}\right)_{i}\left(d^{\beta \alpha}\right)_{i}
$$

Introducing the viscous potential $\Pi_{v}$ as

$$
\Pi_{v}=\frac{-\sum_{i} V_{i} \phi_{i}}{2}
$$

it can be readily shown that $\Pi_{v}$ with the velocity gradients expressed by Eq. (46) is a homogeneous function of degree $k=2$ with respect to the variables $v_{i}^{\alpha}(\alpha=1,2,3 ; i=1, \ldots, n)$. Again, according to the Euler's homogeneous function theorem, Eq. (53) can be rewritten as

$$
\sum_{i} v_{i}^{\alpha}\left[F_{i}^{\alpha}-\frac{\partial \Pi_{v}}{\partial v_{i}^{\alpha}}\right]=0
$$


From the above equation, a suitable form of $F_{i}^{\alpha}$ is obtained as

$$
F_{i}^{\alpha}=\frac{\partial \Pi_{v}}{\partial v_{i}^{\alpha}}
$$

which conserves the total energy. After some algebra, we have

$$
F_{i}^{\alpha}=\sum_{j} V_{i} \tau_{i}^{\alpha \beta} C_{i j}^{\beta}-\sum_{j} V_{j} \tau_{j}^{\alpha \beta} C_{j i}^{\beta}
$$

To summarize, a new set of discrete hydrodynamics equations for evolving density and momentum is obtained as

$$
\dot{\rho}_{i}=\rho_{i} \sum_{j}\left(v_{i}^{\beta}-v_{j}^{\beta}\right) C_{i j}^{\beta}
$$

and

$$
\dot{v}_{i}^{\alpha}=\frac{1}{m_{i}}\left(\sum_{j} V_{i} \sigma_{i}^{\alpha \beta} C_{i j}^{\beta}-\sum_{j} V_{j} \sigma_{j}^{\alpha \beta} C_{j i}^{\beta}\right),
$$

where

$$
\sigma^{\alpha \beta}=-p \delta^{\alpha \beta}+\tau^{\alpha \beta}
$$

Compared to the conventional SPH equations [30] which were derived by following some golden rules tailored for the SPH particle approximation and the corrected version of SPH derived by the variational approach [3], the particle hydrodynamics equations derived here using the energy-based approach is more general in the sense that the choice of particle approximation is open, i.e., any particle approximations including a mix of different schemes can be implemented directly in it. Moreover, we will show in the next subsection that the proposed particle hydrodynamics equations with a proper choice of particle approximation conserve the total angular momentum, while the conventional $\mathrm{SPH}$ equations do not conserve it.

\subsection{Conservation of momentum}

In the absence of external forces, the motion of a number of particles must conserve the total linear and angular momentum. In the following we will discuss the condition under which the proposed discrete hydrodynamics equations will meet this requirement.

The total linear momentum of the system of particles is given by

$$
G^{\alpha}=\sum_{i} m_{i} v_{i}^{\alpha}
$$


The rate of change of the total linear momentum is

$$
\dot{G}^{\alpha}=\sum_{i} P_{i}^{\alpha}+\sum_{i} F_{i}^{\alpha}
$$

From Eq. (52), the force exerted on particle $i$ due to pressure gradient can be expressed as the sum of interaction forces between pairs of particles as

$$
P_{i}^{\alpha}=\sum_{j} P_{i j}^{\alpha}
$$

where

$$
P_{i j}^{\alpha}=V_{j} p_{j} C_{j i}^{\alpha}-V_{i} p_{i} C_{i j}^{\alpha} .
$$

It is straightforward to show

$$
\sum_{i} P_{i}^{\alpha}=\sum_{i} \sum_{j} P_{i j}^{\alpha}=0
$$

because the total sum of all interaction pairs vanish as a consequence of the property, $P_{i j}^{\alpha}=-P_{j i}^{\alpha}$. Following the same approach, we can prove

$$
\sum_{i} F_{i}^{\alpha}=0
$$

Hence conservation of linear momentum is guaranteed by these internal forces derived in the previous subsection using the energy-based approach.

The total angular momentum of the system of particles with respect to the origin is given by

$$
\boldsymbol{H}=\sum_{i} \boldsymbol{x}_{i} \times m_{i} \boldsymbol{v}_{i}
$$

Time differentiation of the above equation gives

$$
\dot{\boldsymbol{H}}=\sum_{i} \boldsymbol{x}_{i} \times \boldsymbol{P}_{i}+\sum_{i} \boldsymbol{x}_{i} \times \boldsymbol{F}_{i}
$$

To conserve angular momentum, the total moment of the internal forces about the origin should vanish, that is,

$$
\sum_{i} \boldsymbol{x}_{i} \times \boldsymbol{P}_{i}=0
$$

and

$$
\sum_{i} \boldsymbol{x}_{i} \times \boldsymbol{F}_{i}=0 .
$$

To check whether angular momentum is preserved by the internal forces proposed in the previous subsection, we follow the procedure used by Bonet and 
Lok [3]. First, recall that a rigid body rotation about the origin is described by a set of velocities given in terms of an angular velocity vector $\delta \boldsymbol{w}$ as

$$
\delta \boldsymbol{v}_{i}=\delta \boldsymbol{w} \times \boldsymbol{x}_{i} .
$$

The variation of the potential $\Pi$ with respect to this type of motion is given by

$$
\delta \Pi=\sum_{i} \boldsymbol{P}_{i} \cdot \delta \boldsymbol{v}_{i}=\sum_{i} \boldsymbol{P}_{i} \cdot\left(\delta \boldsymbol{w} \times \boldsymbol{x}_{i}\right)=\delta \boldsymbol{w} \cdot \sum_{i} \boldsymbol{x}_{i} \times \boldsymbol{P}_{i}
$$

It is clear that, if the potential function is invariant $(\delta \Pi=0)$ with respect to the rigid body rotation given by Eq. (73), then the condition given by Eq. (71) must be satisfied due to the arbitrary nature of the angular velocity $\delta \boldsymbol{w}$. In the same way, we can show that the condition (72) is satisfied if the potential $\Pi_{v}$ is invariant with respect to the rigid body rotation. Therefore, it can be concluded that the invariance of the potential functions $\Pi$ and $\Pi_{v}$ with respect to rigid body rotations leads to preservation of angular momentum.

In order to examine whether the potential functions $\Pi$ and $\Pi_{v}$ are invariant with respect to rigid body rotations consider again one such motion defined by

$$
\boldsymbol{v}(\boldsymbol{x})=\boldsymbol{w} \times \boldsymbol{x},
$$

where $\boldsymbol{w}$ is the angular velocity vector with components $\left[w_{x}, w_{y}, w_{z}\right]^{\mathrm{T}}$. By simple derivation, the true gradient of this velocity field is found to be

$$
\nabla \boldsymbol{v}=\left(\begin{array}{ccc}
0 & -w_{z} & w_{y} \\
w_{z} & 0 & -w_{x} \\
-w_{y} & w_{x} & 0
\end{array}\right)
$$

It is obvious that its trace $\nabla \cdot \boldsymbol{v}$ and the rate of strain tensor $\boldsymbol{d}=\left(\nabla \boldsymbol{v}+\nabla \boldsymbol{v}^{T}\right) / 2$ vanish given the skew nature of $\nabla \boldsymbol{v}$. When the particle approximation given by Eq. (46) is used to calculate the gradient of the velocity field, the correct skew tensor is only obtained if the particle approximation scheme is of first-order consistency, i.e, be able to exactly reproduce any linear function and its gradient. Therefore, only when a particle approximation of first-order consistency such as FPM is adopted for the calculation of the velocity gradients, the potential functions $\Pi$ and $\Pi_{v}$ which are functions of $\boldsymbol{d}$ and $\nabla \cdot \boldsymbol{v}$ would be independent of rigid body rotations and hence angular momentum is preserved.

\subsection{Particle hydrodynamics methods}

In this subsection, we present some particle hydrodynamics methods by actualizing the general set of discrete hydrodynamics equations with a given 
particle approximation. For all the methods presented below, the particle positions are moved consistently according to

$$
\frac{d x_{i}^{\alpha}}{d t}=v_{i}^{\alpha}
$$

\subsubsection{Smoothed Particle Hydrodynamics}

A comparison between Eq. (22) and Eq. (46) gives

$$
C_{i j}^{\beta}=\frac{m_{j}}{\rho_{j}} \frac{\partial W_{i j}}{\partial x_{i}^{\beta}} .
$$

By introducing this expression into Eq. (60) and Eq. (61) and exploiting Eq. (20), a commonly used set of SPH equations for evolving density and momentum is recovered, i.e.,

$$
\dot{\rho}_{i}=\rho_{i} \sum_{j} \frac{m_{j}}{\rho_{j}}\left(v_{i}^{\beta}-v_{j}^{\beta}\right) \frac{\partial W_{i j}}{\partial x_{i}^{\beta}}
$$

and

$$
\dot{v}_{i}^{\alpha}=\sum_{j} m_{j} \frac{\sigma_{i}^{\alpha \beta}+\sigma_{j}^{\alpha \beta}}{\rho_{i} \rho_{j}} \frac{\partial W_{i j}}{\partial x_{i}^{\beta}} .
$$

Note that angular momentum is not conserved by the above SPH equations since the particle approximation by Eq. (22) is not first-order consistent, i.e., it does not correctly evaluate the gradient of any linear velocity field.

\subsubsection{Finite Particle Hydrodynamics}

A comparison between Eq. (38) and Eq. (46) gives

$$
C_{i j}^{\beta}=\frac{m_{j}}{\rho_{j}} \frac{\partial \widetilde{W}_{i j}}{\partial x_{i}^{\beta}} .
$$

By introducing this expression into Eq. (60) and Eq. (61), we obtain a FPMbased set of discrete equations for evolving density and momentum as follows:

$$
\dot{\rho}_{i}=\rho_{i} \sum_{j} \frac{m_{j}}{\rho_{j}}\left(v_{i}^{\beta}-v_{j}^{\beta}\right) \frac{\partial \widetilde{W}_{i j}}{\partial x_{i}^{\beta}}
$$

and

$$
\dot{v}_{i}^{\alpha}=\sum_{j} \frac{m_{j} \sigma_{i}^{\alpha \beta}}{\rho_{i} \rho_{j}} \frac{\widetilde{\partial W}_{i j}}{\partial x_{i}^{\beta}}-\sum_{j} \frac{m_{j} \sigma_{j}^{\alpha \beta}}{\rho_{i} \rho_{j}} \frac{\widetilde{\partial W}_{j i}}{\partial x_{j}^{\beta}}
$$


Note that $\frac{\widetilde{\partial W_{j i}}}{\partial \boldsymbol{x}_{j}} \neq-\frac{\widetilde{\partial W_{i j}}}{\partial \boldsymbol{x}_{i}}$. The numerical model derived here by using the corrected particle approximation (FPM) in the energy-based framework established before will be called Finite Particle Hydrodynamics (FPH). Since the particle approximation by FPM restores the first-order consistency, FPH preserves the total angular momentum.

\subsubsection{Coupled Particle Hydrodynamics}

Since FPH involves solving the local matrix equation for each particle, it is computationally more expensive than SPH. This is the price to pay for an improved accuracy. We here propose a coupled approach for the purpose of reducing the computational cost of $\mathrm{FPH}$ while still maintaining a comparable accuracy with FPH. The idea is to use the SPH approximation of velocity gradients for the interior particles and the FPM approximation of velocity gradients for the exterior particles. Practically, in this paper, the following criteria is used to distinguish between interior and exterior particles, i.e, when a particle finds at least one boundary/surface particle within its support domain, it is regarded as a exterior particle, otherwise, it is regarded as a interior particle. Here a particle on the free surface is defined as a surface particle. In particle methods, there are usually three methods to identify surface particles. One straightforward approach is to record the initial surface particles and these surface particles will remain to be surface particles during the entire simulation. Other particles that turn to be surface particles are not taken into consideration. We use this straightforward approach in our implementations. Another approach is to check the density of the particles by the summation approach (see, for example, Shao and Lo [42]). Since there is no particle outside the surface particles, the approximated densities for the surface particles will be less than the reference value. If the density loss of a particular particle is bigger than a predefined tolerance value, this particle can be regarded as surface particle. This approach seems more effective, though the tolerance value is empirical. Dilts [12] proposed a purely geometric two-dimensional boundary detection algorithm which can detect the boundary particles exactly.

When such an idea of mixed interpolation is adopted within the energy-based framework, we obtain a coupled numerical model for evolving density and momentum as follows:

$$
\dot{\rho}_{i}=\rho_{i} \sum_{j}\left(v_{i}^{\beta}-v_{j}^{\beta}\right) C_{i j}^{\beta}
$$

and

$$
\dot{v}_{i}^{\alpha}=\frac{1}{m_{i}}\left(\sum_{j} V_{i} \sigma_{i}^{\alpha \beta} C_{i j}^{\beta}-\sum_{j} V_{j} \sigma_{j}^{\alpha \beta} C_{j i}^{\beta}\right),
$$


where

$$
C_{i j}^{\beta}= \begin{cases}\frac{m_{j}}{\rho_{j}} \frac{\partial W_{i j}}{\partial x_{i}^{\beta}}, & i \in \text { interior particles; } \\ \frac{m_{j}}{\rho_{j}} \frac{\partial W_{i j}}{\partial x_{i}^{\beta}}, & i \in \text { exterior particles }\end{cases}
$$

and

$$
C_{j i}^{\beta}= \begin{cases}\frac{m_{i}}{\rho_{i}} \frac{\partial W_{j i}}{\partial x_{j}^{\beta}}, & j \in \text { interior particles; } \\ \frac{m_{i}}{\rho_{i}} \frac{\partial W_{j i}}{\partial x_{j}^{\beta}}, & j \in \text { exterior particles. }\end{cases}
$$

The coupled numerical model proposed here will be called Coupled Particle Hydrodynamics $(\mathrm{CPH})$. In principle, $\mathrm{CPH}$ still preserves the linear momentum, but does not conserve the total angular momentum exactly due to the retaining of the $\mathrm{SPH}$ formula for the interior particles. In $\mathrm{CPH}$, the local matrix equations are only solved for the exterior particles, which is normally a small portion of the total particles, hence the computational cost is significantly reduced.

\subsection{Time integration scheme}

To solve the system of ordinary differential equations (Eq. (77), Eq. (79) and Eq. (80) for example) along the particle paths, a simple predictor-corrector scheme [31] is adopted. If $\boldsymbol{X}$ represents the vector of variables $\left(\boldsymbol{x}_{i}, \rho_{i}, \boldsymbol{v}_{i}, S_{i}^{\alpha \beta}\right)$ and $\boldsymbol{F}$ the vector of right-hand sides of the equations., the predictor step predicts the midpoint value of $\boldsymbol{X}$ so that, with time step $\Delta t$, the midpoint $\boldsymbol{X}^{n+1 / 2}$ is

$$
\boldsymbol{X}^{n+1 / 2}=\boldsymbol{X}^{n}+\boldsymbol{F}^{n} \Delta t / 2 .
$$

Then in the corrector step, the value of $\boldsymbol{X}$ at the end of one time step is given by

$$
\boldsymbol{X}^{n+1}=\boldsymbol{X}^{n}+\boldsymbol{F}^{n+1 / 2} \Delta t .
$$

For numerical stability, several time step constraints must be satisfied, including a Courant-Friedrichs-Lewy (CFL) condition,

$$
\Delta t \leq \frac{h}{c}
$$

and additional constraints due to the hydrodynamical force acting on the particle $F_{a}$,

$$
\Delta t \leq \min _{\forall a}\left(\frac{h}{F_{a}}\right)^{1 / 2}
$$

and viscous diffusion,

$$
\Delta t \leq 0.5 \frac{h^{2}}{\nu}
$$


where $\nu=\eta / \rho_{0}$ is the kinematic viscosity. Eq. (90) states that the maximum speed of numerical propagation must exceed the speed of sound $c$. Eq. (91) is based upon the requirement that the time step is always smaller than the inverse Einstein frequency of the system. Eq. (92) comes from the usual condition for an explicit finite difference method for simulating diffusion. At sufficiently high resolution (sufficiently small $h$ ) or large viscosity, Eq. (92) is the dominant constraint for the time step.

\section{Numerical examples}

In this section, three numerical examples concerning free deformation of a viscous fluid patch with free surface are presented to test the performance of the FPH method and the $\mathrm{CPH}$ method proposed in this paper to simulate free surface flows of viscous fluids. A common feature of these examples is that there are no solid boundaries. This allows us to focus on the analysis of accuracy and stability of the proposed methods without complications caused by the presence of solid boundaries. Various ways of implementing the solid boundary conditions have been reported in the past. Yet, it is still a challenging topic in the SPH community.

Kinematic and dynamic conditions are usually required to be satisfied on free surface boundary. Due to their Lagrangian property, the SPH, FPH , and CPH methods implicitly fulfills the kinematic condition. The dynamic condition imposes an appropriate pressure on the free surface. In practice, no additional treatment is made in SPH to impose such a condition. Nevertheless, it is easy to show that SPH automatically enforces a zero-pressure on the free surface thanks to the surface integral in Eq. (15), which is neglected in the final SPH approximation for function derivatives. However, it is not clear whether this is also true for FPH and CPH. Practically, we could have two choices: either making no additional treatment for the dynamics condition like SPH or enforcing zero-pressure on the surface particles. Note that, in the former choice, the pressure on the surface particles is still obtained from the equation of state using the density calculated from the continuity equation like all the other particles. In the following, we will only present the results using the former choice since it gives smoother appearance of the free surface than the latter does and the differences between the two choices are not significant in other aspects. 


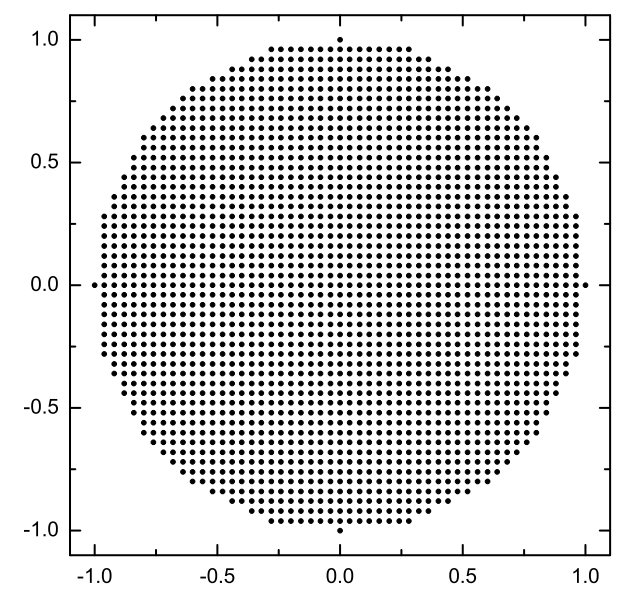

Fig. 1. The initial particle positions of the coarse grid.

\subsection{Drop stretching}

Two-dimensional simulation of the stretching of an initially circular water drop is presented in this section. This example has been used already in the literature of SPH methods $[3,31]$ since an analytical solution is available for comparison [9]. We here consider a drop of a viscous fluid with the viscosity $\eta=10^{-3} \mathrm{kgm}^{-1} \mathrm{~s}^{-1}$. Eq. (8) is adopted as the equation of state with the speed of sound $c=1400 \mathrm{~m} / \mathrm{s}$ and the reference density $\rho_{0}=10^{3} \mathrm{~kg} / \mathrm{m}^{3}$.

The initial geometry of the water drop is a circle of radius $R=1 \mathrm{~m}$ with its center located at the origin $(x=0, y=0)$. There is no external forces but a initial velocity field as $v_{0}^{x}=-A_{0} x, v_{0}^{y}=A_{0} y$ with $A_{0}=100 \mathrm{~s}^{-1}$ and a initial pressure field

$$
p_{0}=\frac{1}{2} \rho_{0} A_{0}^{2}\left[R^{2}-\left(x^{2}+y^{2}\right)\right] .
$$

The latter satisfies a Poisson equation for the pressure ensuring that the fluid is incompressible. The particles comprising the water drop were initially set on our Cartesian grid and those particles within the circle defining the circumference were retained. As a result the drop has a slightly roughened appearance. To check the convergence of the methods, two grids $50 \times 50$ and $100 \times 100$ were used with the number of particles actually involved in the simulations equal to 1961 and 7845 respectively. The coarse grid gives an initial nearest neighbor separation $\Delta d=0.04 \mathrm{~m}$, while the refined grid gives $\Delta d=0.02 \mathrm{~m}$. The initial particle positions of the coarse grid is shown in Fig. 1. The "linked-list" algorithm [20] for searching the nearest neighbor particles (within the distance of $3 h$ ) was adopted with the smoothing length $h=1.3 \Delta d$. A time step of $1 \times 10^{-6} \mathrm{~s}$ was used to ensure numerical stability. No significant differences were observed between the results using the two grids except that the pressure histories predicted by the SPH method using the coarse grid show more pronounced oscillations. In the following, the results using the refined grid are presented. Otherwise, it is indicated explicitly. 


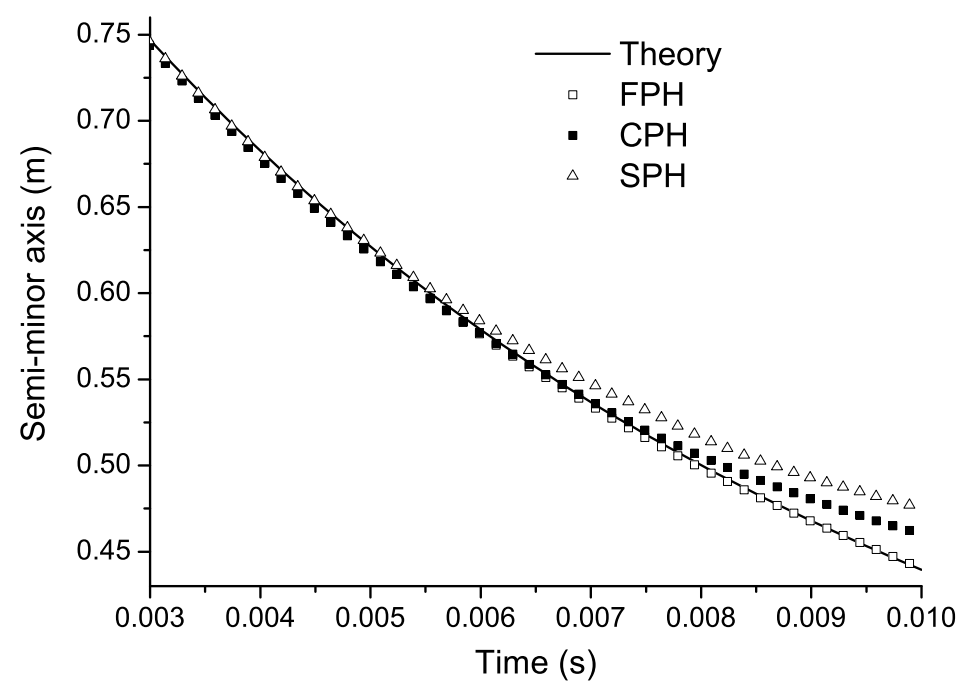

Fig. 2. Numerical and theoretical solutions of $a$ varying with time.

During the calculation the drop should remain elliptical and the value of $a b$ (semi-minor axis times semi-major axis) should remain constant. The solution of $a$ varying with time can be analytically obtained as

$$
\frac{d a}{d t}=-a A
$$

and

$$
\frac{d A}{d t}=\frac{A^{2}\left(a^{4}-\omega^{2}\right)}{a^{4}+\omega^{2}}
$$

where $\omega$ is the initial value of $a b$. The initial conditions are $a(0)=R$ and $A(0)=A_{0}$. The expression of the velocity field is $v^{x}=-A(t) x$ and $v^{y}=A(t) y$. The solution for the pressure is

$$
p(x, y, t)=\frac{1}{2} \rho_{0}\left[\dot{A}\left(x^{2}-y^{2}\right)-A^{2}\left(x^{2}+y^{2}\right)-a^{2}\left(\dot{A}-A^{2}\right)\right] .
$$

A detailed derivation of the above analytical expressions can be found in the $\mathrm{PhD}$ thesis of Colagrossi [9]. Although the derivation given in the thesis is for a non-viscous fluid. It is straightforward to show that the derivation is also valid for a viscous fluid since the velocity field remains linear in space hence the viscous contribution is zero. Eq. (94) and Eq. (95) can be solved together to high accuracy by standard numerical methods such as a RungeKutta method. Here a fifth-order Runge-Kutta method with adaptive time step is used. Once $A(t)$ and $a(t)$ are known, the velocity and pressure fields at any time can also be evaluated.

In Fig. 2 we compare the solutions of $a$ varying with time obtained by different numerical methods and the theory. It turns out that the accuracy of FPH is better than that of $\mathrm{CPH}$, which is better than the accuracy of SPH. The results obtained using FPH is very close to the theory. The maximum errors 


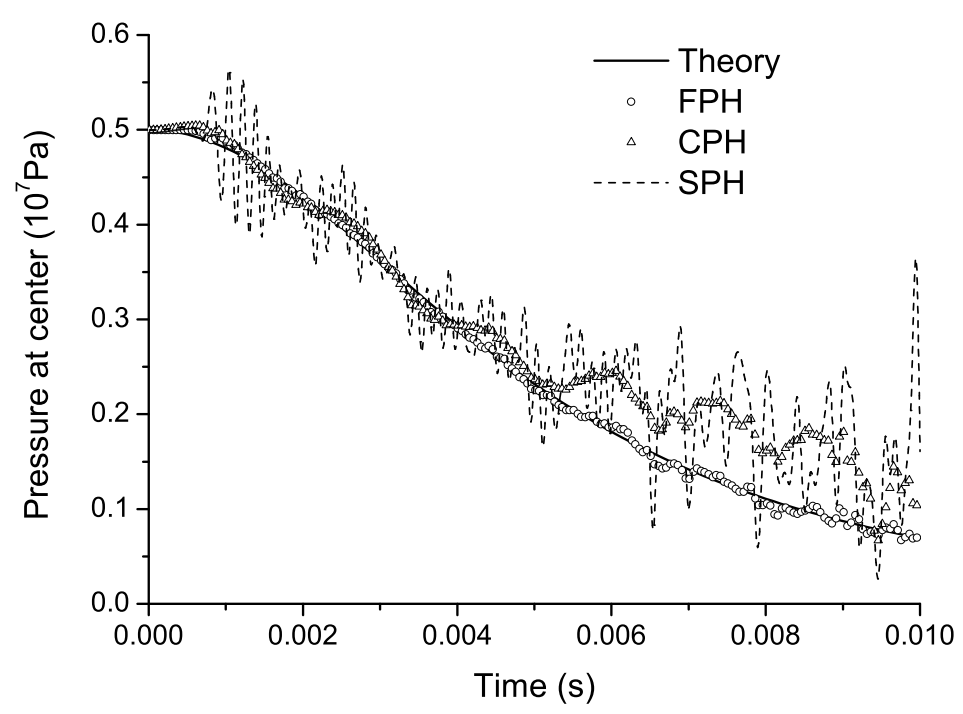

Fig. 3. Numerical and theoretical predictions of the pressure history at the center of the drop.

for $\mathrm{FPH}, \mathrm{CPH}$ and $\mathrm{SPH}$ compared to the theory are less than 1\%,5\% and $10 \%$, respectively.

The pressure history at the center of the drop is shown in Fig. 3. The pressure history predicted by SPH shows pronounced non-physical oscillations, while those predicted by FPH and $\mathrm{CPH}$ show much less oscillations. Again, the pressure history obtained by FPH is very close to the theory. Nevertheless, FPH still produces a small amplitude of pressure oscillation which is due to the weakly compressible approximation used in the method. The results presented here support the conjecture [9] that high amplitude of pressure oscillations predicted by SPH are connected to lower-order formula adopted in the $\mathrm{SPH}$ for the pressure gradient.

Fig. 4 plots the particle positions simulated by the three numerical methods using 1961 particles at the time $0.01 \mathrm{~s}$. The overall particle positions obtained by FPH and CPH are more uniformly distributed and the outer surfaces are far smoother than standard SPH method.

The total CPU time for a typical simulation using 1961 particles and running in a Pentium $4 \mathrm{PC}$ for 10000 time steps is 2089s, 2635s, and 3885s for SPH, $\mathrm{CPH}$, and $\mathrm{FPH}$, respectively. $\mathrm{CPH}$ gains a $32 \%$ computational saving relative to FPH. It is expected that the percentage of computational saving by $\mathrm{CPH}$ increases with increasing the total number of particles.

Finally, it is important to note that the results predicted by $\mathrm{SPH}$ and $\mathrm{CPH}$ become worse at longer times (see Fig. 2 and Fig. 3). This is due to the fact that we are using an isotropic kernel with a constant smoothing length $h=1.3 \Delta d$, which corresponds to a particle support domain with a width of 

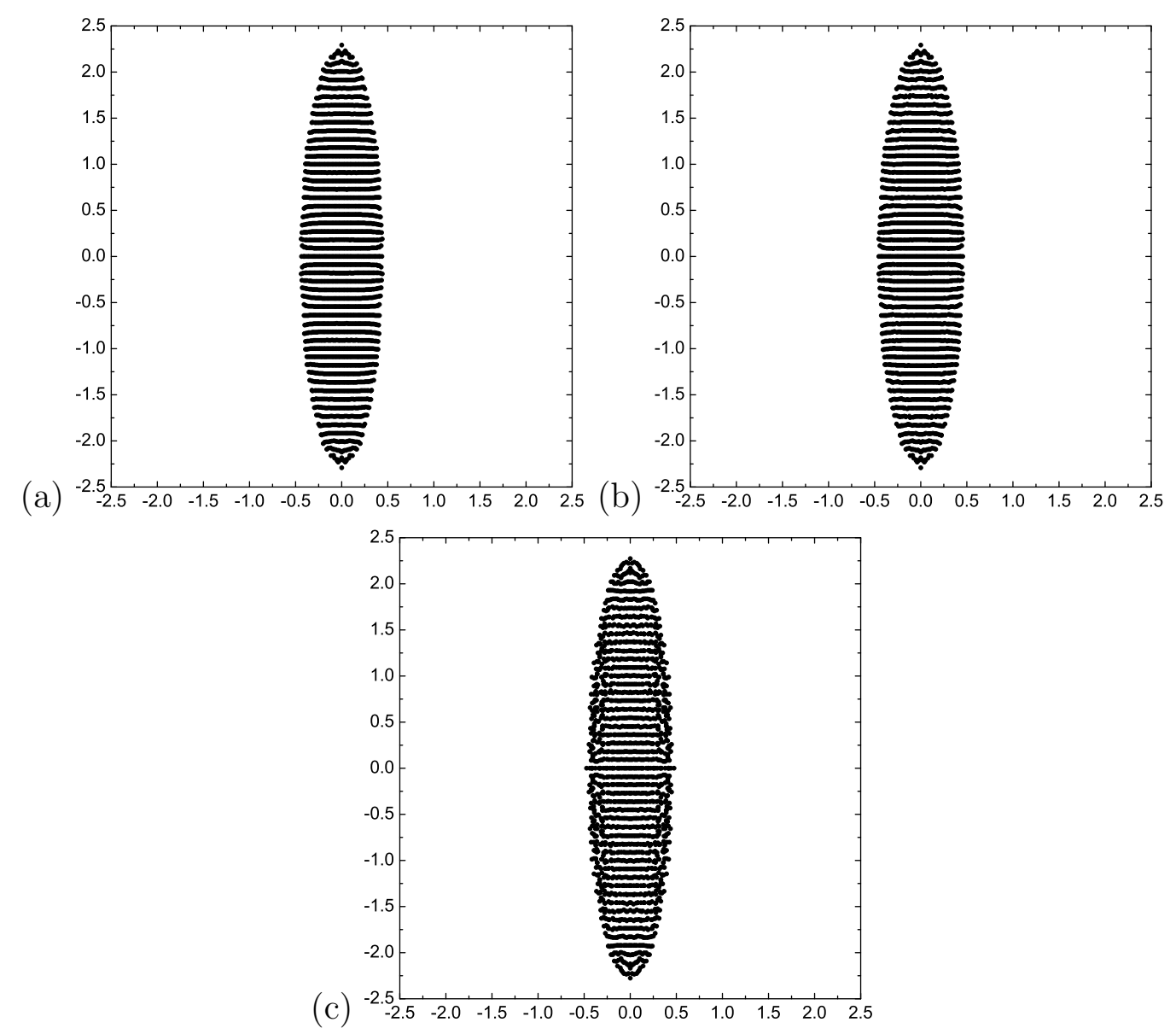

Fig. 4. The positions of 1961 particles at the time 0.01 s predicted by (a) FPH, (b) $\mathrm{CPH}$, and (c) SPH.

$3.9 \Delta d$. At a particular point in time, say at $t=0.008 \mathrm{~s}$, the strain reaches 2 in the $y$ direction. The particle kernel which initially reached 3 layers of neighbors in the $y$ direction can only reach one y-layer of neighbors by the time 0.008s. This is the reason for the accuracy degradation of SPH and CPH simulations: particles undergo so massive amounts of strain that they do not overlap anymore and the particle set loses its regularity. With this in mind, the accuracy of FPH is quite remarkable for these deformations. However, as the simulation goes on and the deformation increases further, FPH will eventually encounter the problem that the matrix (37) becomes ill conditioned. The method proposed in $[40,43]$ may be implemented to solve the problems associated with large deformation. The idea is to replace the isotropic kernel function $W$ defined in Eq. (21) by an adaptive anisotropic kernel function with tensor smoothing lengthes which are not fixed but evolved according to specific equations to follow the mean particle spacing as it varies in time, space, and direction around each particle. The particle regularization technique [4] and the similar one called re-meshing (or re-initialization) [5] can also be considered. Yet, it is an open problem how to design suitable regularization or re-meshing techniques in the presence of free surface as in case of a deforming 


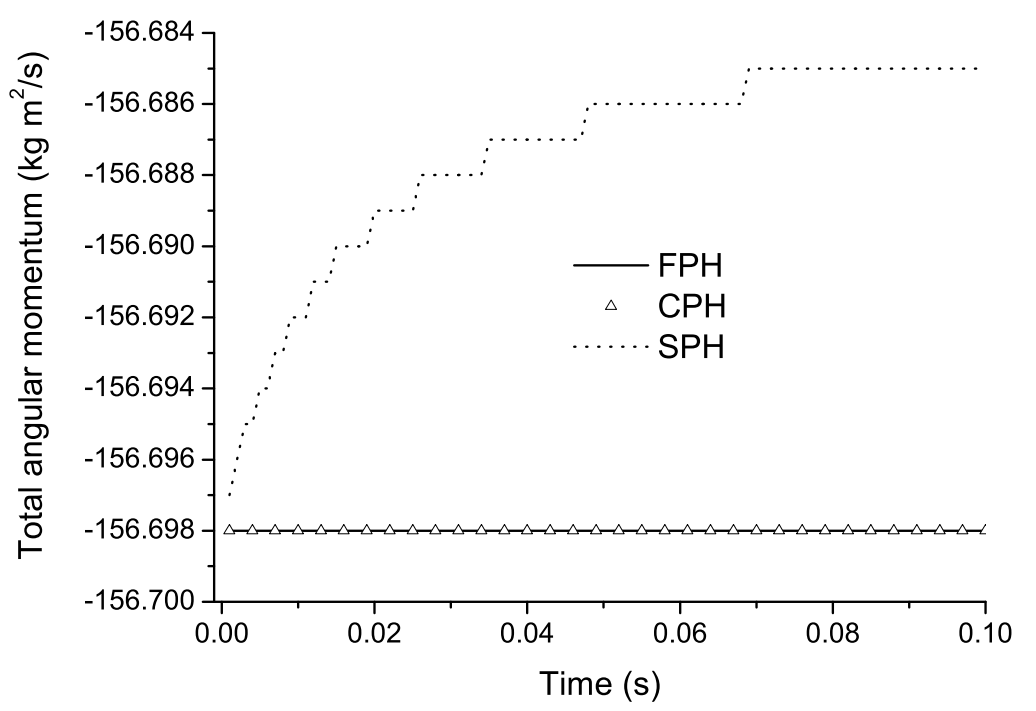

Fig. 5. Total angular momentum with time.

drop.

\subsection{Drop rotating}

In this example we present numerical simulations in the case of a circular drop under rigid body rotation as considered for example in Hoover et al. [21]. The purpose of this example is to check whether the total angular momentum is preserved by the three methods studied in this paper and verify the corresponding theoretical argument in sec. 3.2.

The initial geometry of the drop is exactly the same as in sec. 4.1 and an initial velocity field of $v_{0}^{x}=0.1 y, v_{0}^{y}=-0.1 x$ representing a constant rigid body rotation is assigned. The initial pressure is set to zero everywhere. A high viscosity $\eta=500 \mathrm{kgm}^{-1} \mathrm{~s}^{-1}$ is used. Eq. (8) is again adopted as the equation of state with the speed of sound $c=14 \mathrm{~m} / \mathrm{s}$ and the reference density $\rho_{0}=10^{3} \mathrm{~kg} / \mathrm{m}^{3}$. The space discretization uses a total of 1961 particles whose initial positions are generated from a coarse grid as described in sec. 4.1. The initial nearest neighbor separation is $\Delta d=0.04 \mathrm{~m}$. As in sec. 4.1, the smoothing length $h$ is set to $1.3 \Delta d$. A constant time step of $1 \times 10^{-5} \mathrm{~s}$ is used.

Fig. 5 shows the total angular momentum with time. From the figure, it is seen that the total angular momentum is conserved by FPH method, but not by SPH method. This verifies the theoretical argument in sec. 3.2. Theoretically, $\mathrm{CPH}$ method should not preserve the total angular momentum due to the loss of first-order consistency for the interior particles. However, practically, $\mathrm{CPH}$ preserves the total angular momentum as well as FPH does for the case 
studied here. This supports our thought that it may be sufficient to restore first-order consistency only for the exterior particles. Numerical results (not shown here) also confirm that all the three methods conserve the total linear momentum.

\subsection{Rotation of a square fluid patch}

The third example is about the rotation of a free surface square fluid patch. A 2D patch of viscous fluid is initially square in shape and subjected to the following velocity field: $v_{0}^{x}=\omega y, v_{0}^{y}=-\omega x$, where $\omega$ denotes an arbitrary angular velocity. In this test case, the fluid patch undergoes large free boundary deformations, which are responsible for the occurrence of strong instabilities. This challenging test for the SPH methods was first proposed and studied by Colagrossi [9]. In our simulations, the initial square is $2 \mathrm{~m}$ long each side and centered at $(x=0, y=0), \omega$ is set to $100 \mathrm{~s}^{-1}$, the initial pressure field is taken as zero everywhere, though an analytical incompressible solution of the Poisson equation exists [9], the fluid viscosity is $\eta=0.5 \mathrm{kgm}^{-1} \mathrm{~s}^{-1}$, the equation of state with the sound speed and the reference density is the same as in the first example. A total of $2601(51 \times 51)$ particles with $\Delta d=0.04 \mathrm{~m}$ and $h=1.3 \Delta d$ and a constant time step of $1 \times 10^{-6} \mathrm{~s}$ are used in the simulations.

Fig. 6 and Fig. 7 show the particle positions at $t=0.015 \mathrm{~s}$ and $t=0.02 \mathrm{~s}$ respectively. The results are qualitatively in agreement with those obtained by Colagrossi [9]. Due to centrifugal forces, the four corners of the fluid patch are stretched and finally develop into four arms. Meanwhile, the square size of the patch decreases. At the time $t=0.015 \mathrm{~s}$, unrealistic fluid fragmentation due to particle clumping is observed in the results by SPH, but does not appear yet in the results by $\mathrm{CPH}$ and $\mathrm{FPH}$. At a latter time $t=0.02$ $\mathrm{s}$, fragmentation is further enlarged in the results by SPH, becomes obvious in the results by $\mathrm{CPH}$, and starts to show a little bit in the results by FPH. These numerical fractures progressively degrade the interpolation procedure and deteriorate the entire simulation. The phenomena is not unique in the case simulated here, but a common one reported in many applications of SPH. The reason behind is the so-called tensile instability [44], which states that, when the material is in a state of tensile stress (negative pressure for example), particles start to form clumps and the motion of the particles becomes unstable. From the results presented here, we can see that tensile instability is closely related to the low-order formula for the pressure gradient approximation and it can be greatly reduced by using higher order variants of SPH such as FPH and $\mathrm{CPH}$. However, neither $\mathrm{FPH}$ nor $\mathrm{CPH}$ removes completely the problem of tensile instability. Monaghan proposed an artificial stress term for the momentum equation [33]. This prevents particle clumping, but unfortunately contaminates the simulation with induced chaotic pressure and velocity 

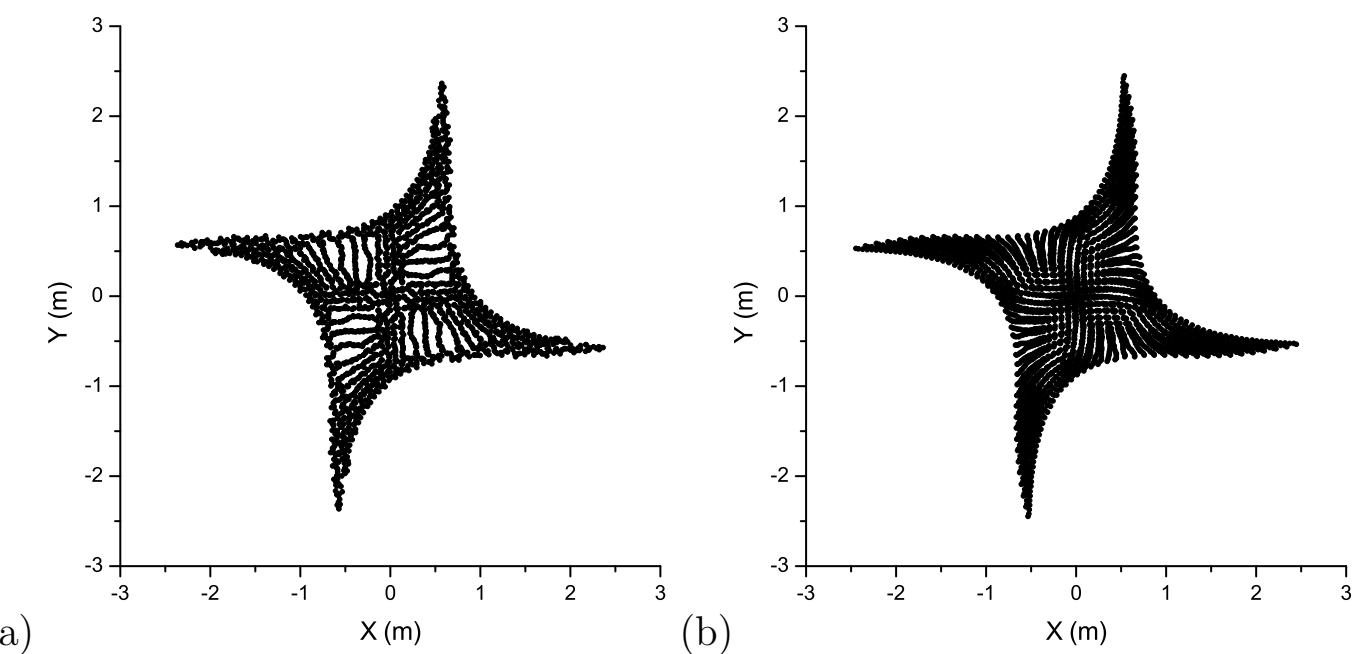

(a)

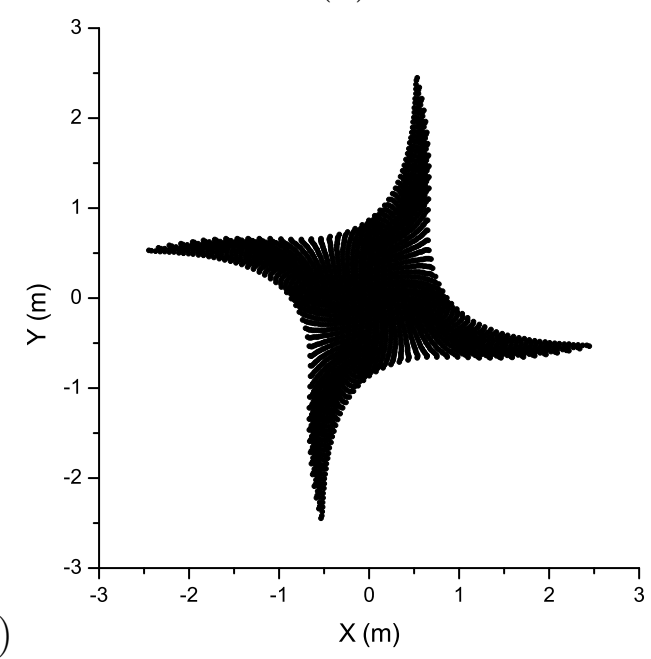

Fig. 6. The positions of 2601 particles at the time 0.015 s predicted by (a) SPH, (b) $\mathrm{CPH}$, and (c) FPH.

distributions.

It is known that SPH methods can be stabilized using artificial viscosity [30]. We here test its effects on FPH and CPH. An artificial viscosity term is added to the discrete momentum equation as usually done for SPH methods. This term is given as

$$
\Pi_{i j}=\frac{-\alpha_{\Pi} \bar{c}_{i j} \phi_{i j}+\beta_{\Pi} \phi_{i j}^{2}}{\bar{\rho}_{i j}} \quad \text { for } \quad \boldsymbol{v}_{i j} \cdot \boldsymbol{x}_{i j}<0,
$$

and

$$
\Pi_{i j}=0 \quad \text { for } \quad \boldsymbol{v}_{i j} \cdot \boldsymbol{x}_{i j} \geq 0
$$

where

$$
\begin{gathered}
\phi_{i j}=\frac{h \boldsymbol{v}_{i j} \cdot \boldsymbol{x}_{i j}}{\left|\boldsymbol{x}_{i j}\right|^{2}+0.01 h^{2}}, \\
\bar{c}_{i j}=\left(c_{i}+c_{j}\right) / 2,
\end{gathered}
$$



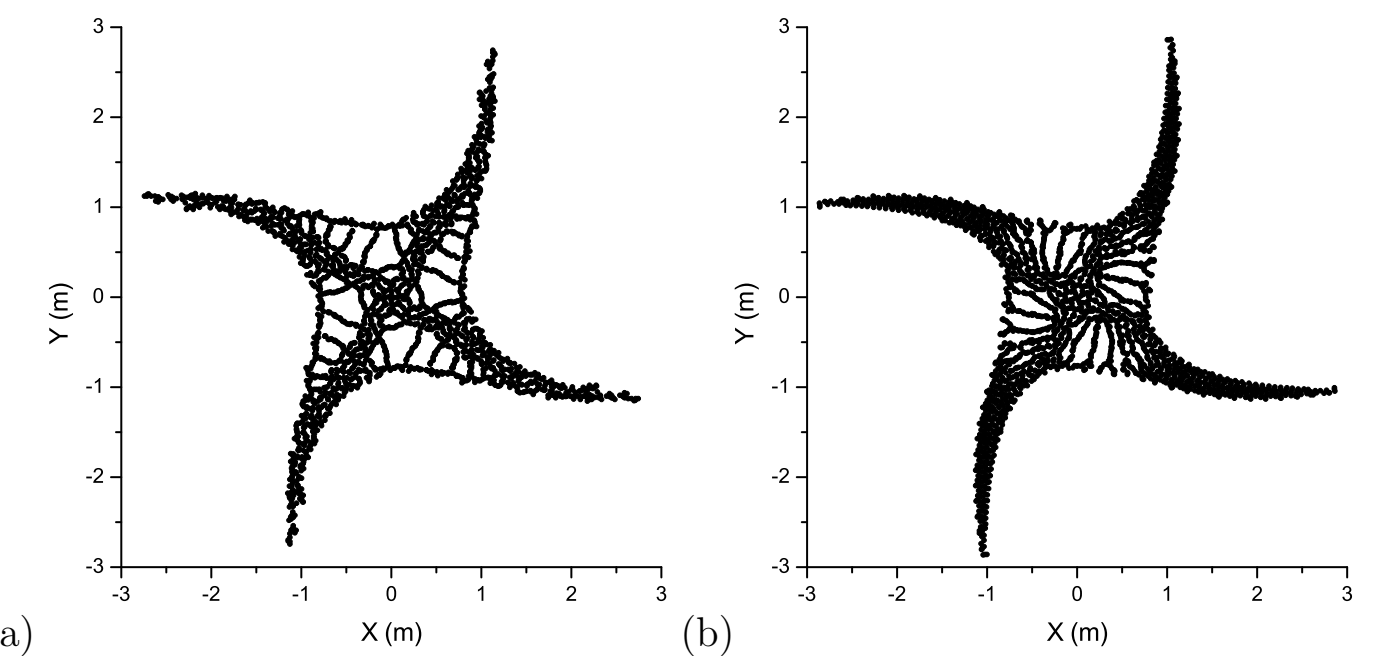

(a)

(b)

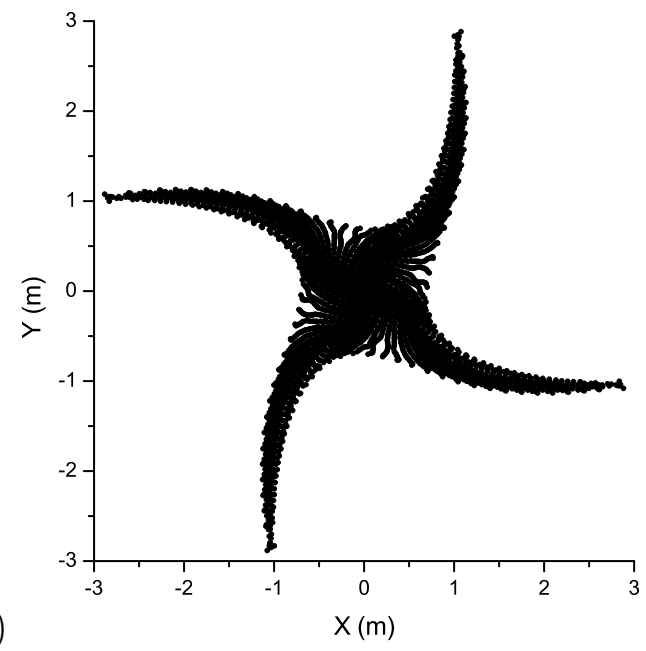

Fig. 7. The positions of 2601 particles at the time 0.02 s predicted by (a) SPH, (b) $\mathrm{CPH}$, and (c) FPH.

$$
\begin{gathered}
\bar{\rho}_{i j}=\left(\rho_{i}+\rho_{j}\right) / 2, \\
\boldsymbol{v}_{i j}=\boldsymbol{v}_{i}-\boldsymbol{v}_{j} \\
\boldsymbol{x}_{i j}=\boldsymbol{x}_{i}-\boldsymbol{x}_{j} .
\end{gathered}
$$

In the above expressions, $\alpha_{\Pi}$ and $\beta_{\Pi}$ are constants that are all typically set to 1 approximately. The $0.01 h^{2}$ term is included to prevent numerical divergence when two particles are approaching each other. Fig. 8 presents the results with $\alpha_{\Pi}=0.1$ and $\beta_{\Pi}=0.2$ at $t=0.02 \mathrm{~s}$. It is found that, with this small added artificial viscosity, unrealistic fragmentation is almost gone for $\mathrm{FPH}$ and $\mathrm{CPH}$ and dramatically reduced for SPH. 

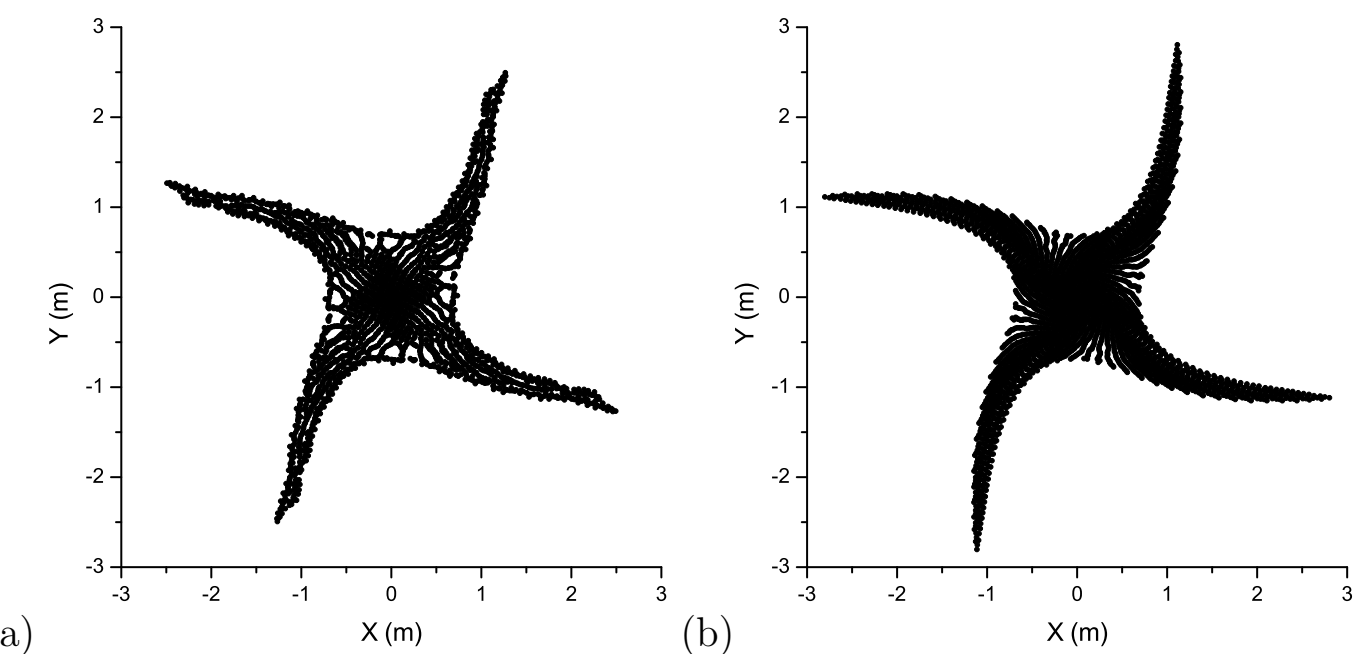

(a)

(b)

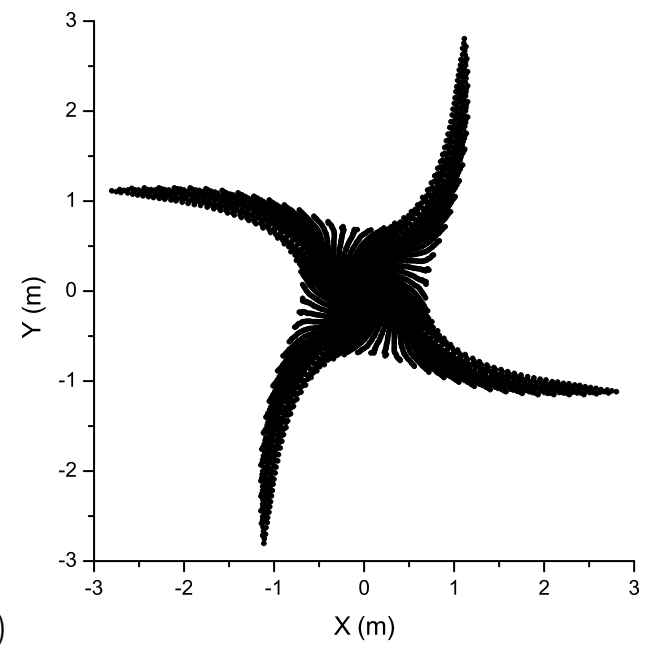

(c)

Fig. 8. The positions of 2601 particles at the time $0.02 \mathrm{~s}$ predicted by (a) SPH, (b) $\mathrm{CPH}$, and (c) FPH. Artificial viscosity is used with $\alpha_{\Pi}=0.1$ and $\beta_{\Pi}=0.2$.

\section{Conclusions}

Enhancement of the smoothed particle hydrodynamics (SPH) method for the simulation of free surface viscous flows has been considered in this paper. We have applied an energy-based framework to derive a general set of discrete hydrodynamics equations which conserves the total linear momentum for any particle approximations and the total angular momentum for particle approximations of first-order consistency. Two new methods, the finite particle hydrodynamics (FPH) method and the coupled particle hydrodynamics $(\mathrm{CPH})$ method, have been proposed within the framework. In FPH, finite particle method (FPM) is used to generate the corrected particle approximation which has first-order consistency. In CPH, a coupled FPM/SPH particle approximation is adopted. In terms of accuracy and stability, both FPH and $\mathrm{CPH}$ outperform the SPH method in the three test cases of free deformations of fluid patches with free surfaces. It is shown that $\mathrm{CPH}$ is computationally 
more efficient than FPH.

\section{Acknowledgements}

This research work has been partially supported by the Swiss National Science Foundation, grant number 200021-105193. The authors would like to thank the two anonymous referees for giving very helpful comments and suggestions that have greatly improved this paper.

\section{References}

[1] R. Ata, A. Soulaïmani, A stabilized SPH method for inviscid shallow water flows, Int. J. Numer. Meth. Fluids 47 (2005) 139-159.

[2] W. Benz, E. Asphaug, Simulations of brittle solids using smooth particle hydrodynamics, Comput. Phys. Commun. 87 (1995) 253-265.

[3] J. Bonet, T.-S.L. Lok, Variational and momentum preservation aspects of smooth particle hydrodynamic formulations, Comput. Methods Appl. Mech. Engrg. 180 (1999) 97-115.

[4] S. Børve, M. Omang, J. Trulsen, Regularized smoothed particle hydrodynamics: A new approach to simulating magnetohydrodynamic shocks, Astrophys. J. 561 (2001) 82-93.

[5] A. Chaniotis, D. Poulikakos, P. Koumoutsakos, Remeshed smoothed particle hydrodynamics for the simulation of viscous and heat conducting flows, J. Comput. Phys. 182 (2002) 67-90.

[6] J.K. Chen, J.E. Beraun, A generalized smoothed particle hydrodynamics method for nonlinear dynamic problems, Comput. Methods Appl. Mech. Engrg. 190 (2000) 225-239.

[7] P.W. Cleary, J.J. Monaghan, Conduction modeling using smoothed particle hydrodynamics, J. Comput. Phys. 148 (1999) 227-264.

[8] P.W. Cleary, M. Prakash, Discrete-element modelling and smoothed particle hydrodynamics: potential in the environmental sciences, Phil. Trans. R. Soc. Lond. A 362 (2004) 2003-2030.

[9] A. Colagrossi, A meshless Lagrangian method for free-surface and interface flows with fragmentation, Ph.D. thesis, Università di Roma La Sapienza, 2005.

[10] S.J. Cummins, M. Rudman, An SPH Projection Method, J. Comput. Phys. 152 (1999) 584-607. 
[11] G.A. Dilts, Moving least square particle hydrodynamics I: consistency and stability, Int. J. Numer. Methods Engng. 44 (1999) 1115-1155.

[12] G.A. Dilts, Moving least-squares particle hydrodynamics II: Conservation and boundaries, Int. J. Numer. Methods Engng. 48 (2000) 1503-1524.

[13] M. Ellero, M. Kröger, S. Hess, Viscoelastic flows studied by smoothed particle dynamics, J. Non-Newtonian Fluid Mech. 105 (2002) 35-51.

[14] M. Ellero, R.I. Tanner, SPH simulations of transient viscoelastic flows at low Reynolds number, J. Non-Newtonian Fluid Mech. 132 (2005) 61-72.

[15] P. Español, M. Revenga, Smoothed dissipative particle dynamics, Physical Review E 67 (2003) 267051-2670512.

[16] J. Fang, R.G. Owens, L. Tacher, A. Parriaux, A numerical study of the SPH method for simulating transient viscoelastic free surface flows, J. NonNewtonian Fluid Mech. 139 (2006) 68-84.

[17] O. Flebbe, S. Munzel, H. Herold, H. Riffert, H. Ruder, Smoothed particle hydrodynamics-physical viscosity and the simulation of accretion disks, Astrophys. J. 431 (1994) 754-760.

[18] R.A. Gingold, J.J. Monaghan, Smoothed particle hydrodynamics theory and application to non-spherical stars, Mon. Not. R. Astron. Soc. 181 (1977) 375389.

[19] M. Grmela, H.C. Öttinger, Dynamics and thermodynamics of complex fluids. I. Development of a general formalism, Phys. Rev. E 56 (1997) 6620-6632.

[20] R.W. Hockney, J.W. Eastwood, Computer Simulation Using Particles, McGraw-Hill, New York, 1981.

[21] W.G. Hoover, C.G. Hoover, E.C. Merritt, Smooth particle applied mechanics: conservation of angular momentum with tensile stability and velocity averaging, Phys. Rev. E 69 (2004) 016702-1-10.

[22] G.R. Johnson, S.R. Beissel, Normalised smoothing functions for SPH impact computations, Int. J. Numer. Methods Engng. 39 (1996) 2725-2741.

[23] L.D. Libersky, A.G. Petschek, Smooth particle hydrodynamics with strength of materials, in: H.E. Trease, M.J. Fritts, W.P. Crowley (Eds.), Advances in the Free-Lagrange Method, Moran, WY, USA, June 1990, vol. 395, Springer-Verlag, New York/Berlin, 1991, p. 248-257 (Lect. Notes in Phys.).

[24] L.D. Libersky, A.G. Petschek, T.C. Carney, J.R. Hipp, F.A. Allahdadi, High strain Lagrangian hydrodynamics, J. Comput. Phys. 109 (1993) 67-75.

[25] G.R. Liu, M.B. Liu, Smoothed Particle Hydrodynamics-A Meshfree Particle Method, World Scientific, 2003.

[26] M.B. Liu, W.P. Xie, G.R. Liu, Modeling incompressible flows using a finite particle method, Appl. Math. Model. 29 (2005) 1252-1270. 
[27] W.K. Liu, S. Jun, Y.F. Zhang, Reproducing kernel particle methods, Int. J. Numer. Methods Fl. 20 (1995) 1081-1106.

[28] L.B. Lucy, A numerical approach to the testing of the fission hypothesis, Astron. J. 83 (1977) 1013-1024.

[29] C. Maveyraud, W. Benz, A. Sornette, D. Sornette, Solid friction at high sliding velocities: an explicit three-dimensional dynamical smoothed particle hydrodynamics approach, J. Geophys. Res. 104 (1999) 28769-28788.

[30] J.J. Monaghan, Smoothed particle hydrodynamics, Annu. Rev. Astron. Astrophys. 30 (1992) 543-574.

[31] J.J. Monaghan, Simulating free surface flows with SPH, J. Comput. Phys. 110 (1994) 399-406.

[32] J.J. Monaghan, A. Kocharyan, SPH simulation of multi-phase flow, Comput. Phys. Commun. 87 (1995) 225-235.

[33] J.J. Monaghan, SPH without a tensile instability, J. Comput. Phys. 159 (2000) 290-311.

[34] J.J. Monaghan, SPH compressible turbulence, Mon. Not. R. Astron. Soc. 335 (2002) 843-852.

[35] J.J. Monaghan, Smoothed particle hydrodynamics, Rep. Prog. Phys. 68 (2005) 1703-1759.

[36] J.P. Morris, P.J. Fox, Y. Zhu, Modeling low Reynolds number incompressible flows using SPH, J. Comput. Phys. 136 (1997) 214-226.

[37] J.P. Morris, Simulating surface tension with smoothed particle hydrodynamics, Int. J. Numer. Meth. Fluids 33 (2000) 333-353.

[38] L. Oger, S.B. Savage, Smoothed particle hydrodynamics for cohesive grains, Comput. Meth. Appl. Mech. Engrg. 180 (1999) 169-183.

[39] H.C. Öttinger, M. Grmela, Dynamics and thermodynamics of complex fluids. II. Illustrations of a general formalism, Phys. Rev. E 56 (1997) 6633-6655.

[40] J.M. Owen, J.V. Villumsen, P.R. Shapiro, H. Martel, Adaptive smoothed particle hydrodynamics: Methodology. II., Astrophys. J. Suppl. Ser. 116 (1998) 155-209.

[41] P.W. Randles, L.D. Libersky, Smoothed particle hydrodynamics: Some recent improvements and applications, Comput. Methods Appl. Mech. Engrg. 139 (1996) 375-408.

[42] S. Shao, E.Y.M. Lo, Incompressible SPH method for simulating Newtonian and non-Newtonian flows with a free surface, Advances Water Resour. 26 (7) (2003) $787-800$.

[43] P.R. Shapiro, H. Martel, J.V. Villumsen, J.M. Owen, Adaptive smoothed particle hydrodynamics, with application to cosmology: Methodology, Astrophys. J. Suppl. Ser. 103 (1996) 269-330. 
[44] J.W. Swegle, D.L. Hicks, S.W. Attaway, Smoothed particle hydrodynamics stability analysis, J. Comput. Phys. 116 (1995) 123-134.

[45] H. Takeda, S.M. Miyama, M. Sekiya, Numerical simulation of viscous flow by smoothed particle hydrodynamics, Prog. Theor. Phys. 92 (5) (1994) 939-959.

[46] S.J. Watkins, A.S. Bhattal, N. Francis, J.A. Turner, A.P. Whitworth, A new prescription for viscosity in smoothed particle hydrodynamics, Astron. Astrophys. Suppl. Ser. 119 (1996) 177-187.

[47] G.M. Zhang, R.C. Batra, Modified smoothed particle hydrodynamics method and its applications to transient problems, Comput. Mech. 34 (2004) 137-146. 